\title{
Biocompatible water-soluble endometallofullerenes: peculiarities of self-assembly in aqueous solutions and ordering under an applied magnetic field
}

\author{
V. T. Lebedev, Yu. V. Kulvelis, V. V. Runov, A. A. Szhogina, M. V. Suyasova \\ Petersburg Nuclear Physics Institute, NRC Kurchatov Institute, 188300 Gatchina, \\ Leningrad distr., Russia \\ vlebedev@pnpi.spb.ru,kulvelis@pnpi.spb.ru,runov@pnpi.spb.ru, \\ allin_ok2@mail.ru, marinasuyasova@pnpi.spb.ru
}

\section{PACS 61.48-c; 36.20.-r; 82.35.Np; 61.25.he DOI 10.17586/2220-8054-2016-7-1-22-29}

\begin{abstract}
The self-organization of water-soluble endometallofullerenes (fullerenols) in solutions has been studied by neutron scattering in connection with their applications (Magneto-Resonance Imaging, $X$-Ray Tomography). Their functional characteristics depend strongly on molecular self-assembly which may be altered by chemical additives, concentration increase or the magnetic field applied. Polarized neutrons have been used to search paramagnetic fullerenols' organization into supramolecular structures influenced by the fullerenol concentration and their intensified interactions in a magnetic field.
\end{abstract}

Keywords: endofullerene, structure, assembly, biomedicine, neutron scattering.

Received: 20 November 2015

\section{Introduction}

The application of paramagnetic endometallofullerenes (EMF) [1] such as Gd@ $\mathrm{C}_{82}$ and similar structures in biomedicine (contrasting agents for magnetic resonance imaging (MRI), $X$-Ray tomography, tracers being the isotopes encapsulated in carbon cages etc.) $[2,3]$ is closely related to the study of various water-soluble EMF derivatives in aqueous solutions to understand mechanisms of molecular assembly altered by the functional properties of these substances $[4,5]$.

For example, there is a remarkable well known acceleration of the spin relaxation for protons in the vicinity of paramagnetic EMF molecules in aqueous solutions or biological tissues (e.g. fullerenols $\mathrm{Gd} @ \mathrm{C}_{82}(\mathrm{OH})_{X}$ ) [6]. This effect is enhanced by the association of EMFs and is dependent upon the size and forms of EMF clusters [7]. This proton relaxivity enhancement effect is induced by dissolved EMF molecules and is of great importance to achieve higher resolution in MRI-diagnostics while simultaneously avoiding toxicity issues associated with heavy metals (e.g. Gd), since their atoms are encapsulated in carbon cages and well-protected against chemical interaction with the surrounding media in living organisms. Along with this, a gain in relaxivity can be expected when one uses paramagnetic EMFs mixed with diamagnetic (empty) EMFs, since their labile $\pi$-electrons may be polarized by a neighboring paramagnetic molecule. Thus, one can expect an amplification of fluctuating local fields due to additional effects of proton relaxivity acceleration. However, these inductive effects between paramagnetic and diamagnetic molecules are not yet studied. Additionally, there is only a poor knowledge of the mechanisms for the local ordering of paramagnetic fullerenols in solutions, especially under the influence of applied magnetic fields which can be relatively strong (few Tesla in MRI-tests).

It should be noted that a set of effective experimental methods and instruments to comprehensively analyze the self-organization of water-soluble EMFs in aqueous media still 
remains restricted, especially regarding the study of both supramolecular and magnetic ordering in EMF solutions in magnetic fields and by altering other parameters (e.g. concentration of paramagnetic EMFs, $\mathrm{pH}$-factor, temperature).

Among these structural methods (e.g. $X$-ray or light scattering), only the polarized neutron reflectometer is a powerful enough instrument to obtain detailed information on the molecular and magnetic ordering and subtle effects of their interference over a wide range of spatial scales $\left(10^{0}-10^{2} \mathrm{~nm}\right)$. It is of principal importance that the polarized neutron beam does not disturb the sample and gives information on the size and forms of molecular arrangements, as well as the possible magnetic ordering of EMFs under the applied magnetic field. The polarization of scattered neutrons is very sensitive to fine magnetic correlations in the ensembles of paramagnetic objects [8].

The aim of our experiments on aqueous mixtures of paramagnetic and diamagnetic fullerenols is to study the behavior of these systems under magnetic field to discover original and field-induced mechanisms for the self-assembly of fullerenols by varying their concentration and magnetic induction.

\section{Experimental}

\subsection{Samples and measurements}

The samples were synthesized at PNPI for neutron experiments. Mixtures of $\mathrm{Gd} @ \mathrm{C}_{82}(\mathrm{OH})_{X}(X=24-30)$ and $\mathrm{C}_{60}(\mathrm{OH})_{X}$ were prepared keeping a constant level of paramagnetic component $(30 \mathrm{wt} \%)$. These mixtures were dissolved in light water to obtain solutions with concentrations $C=0.044,0.056,1.04$ and $2.0 \mathrm{wt} \%$ (Samples 1-4) where the upper limit is close the threshold for fullerenol solubility $\left(C_{\max }=2.3 \mathrm{wt} \%\right)$.

Polarized small-angle neutron scattering experiments (PSANS) have been carried out at ambient temperature on the Diffractometer "Vector" (PNPI) [8] over a range of momentum transfer $q=(4 \pi / \lambda) \sin (\theta / 2)=0.03-0.45 \mathrm{~nm}^{-1}$ where neutron wavelength $\lambda=0.92 \mathrm{~nm}$ $(\Delta \lambda / \lambda=0.25)$, scattering angles $0 \leq \theta \leq 3^{\circ}$. The $q$-interval corresponded to the scales of the fullerenols structures $R \sim 2 \pi / q \sim 10^{1}-10^{2} \mathrm{~nm}$. In the detector plane $(X, Y)$, the scattering was registered with $q$-resolution $\delta q_{X}, \delta q_{Y} \approx 4.5 \cdot 10^{-3} \mathrm{~nm}^{-1}$.

For the Samples 1-4 (solutions layers, thickness $d_{S}=1 \mathrm{~mm}$ ) an isotropic scattering was observed in the external field $B=0.01,0.6,1.0 \mathrm{~T}$ applied along the vertical axis $Y$ (incident beam along $Z$-axis). In scattering measurements, the direction of vertical polarization of the beam $\left(P_{0}=0.94\right)$ was periodically reversed $\left( \pm \mathbf{P}_{\mathbf{0}}\right)$ to detect the intensities $I^{ \pm}(\mathbf{q})$ which were used to calculate the sums $I_{t}=I^{+}+I^{-}$and the differences $\Delta I=I^{+}-I^{-}$sensitive to interference effects of the scattering on molecules (atomic nuclei) and the magnetic moments of $\mathrm{Gd}$ atoms. A measure of such nuclear magnetic interference is the effective polarization $P_{e f}=\Delta I / I_{t}$. The measured intensities $\left(I_{t}\right)$ were used to determine the scattering intensities $\left(I_{S}\right)$ and the related cross sections, taking into account the neutron transmissions for the samples, the background level and the contribution of the beam that passed through the samples without scattering. Finally, the differential cross sections per unit solid angle $(\Omega)$ and $\mathrm{cm}^{3}$ of sample volume were computed in absolute units, $d \sigma(q) / d \Omega=\left(I_{S} / I_{W}\right)\left(d_{W} / d_{S}\right)\left(d \sigma_{W}(q) / d \Omega\right)$, where $I_{W}$ is the intensity measured for $\mathrm{H}_{2} \mathrm{O}$-standard (layer, thickness $d_{W}=1 \mathrm{~mm}$ ) at the same experimental conditions. Here, the cross section of water $d \sigma_{W}(q) / d \Omega=1.089 \mathrm{~cm}^{-1} / \mathrm{cm}^{3}$ is used [9]. In following treatment the notation $\sigma(q)=d \sigma(q) / d \Omega$ is accepted. 


\subsection{Discussion}

In the experiments, the magnetic field was varied from minimum magnitude $B_{1}=0.01 \mathrm{~T}$ (guide field to keep beam polarization) up to intermediate and maximum magnitudes, $B_{2}=$ $0.6 \mathrm{~T}, B_{3}=1.0 \mathrm{~T}$. At these induction values, the measurement was organized in such a way that periodically the induction was switched on and after few hours of data collection it was switched off for the measurement at minimum field $B_{1}$. These weakly magnetic substances did not reveal any significant hysteresis and demonstrated a reversible behavior detected in the scattering. In Fig. 1, the cross sections of the solutions with concentrations $C=0.044-2.0 \mathrm{wt} \%$ are shown as dependent on momentum transfer for minimum and maximum fields.

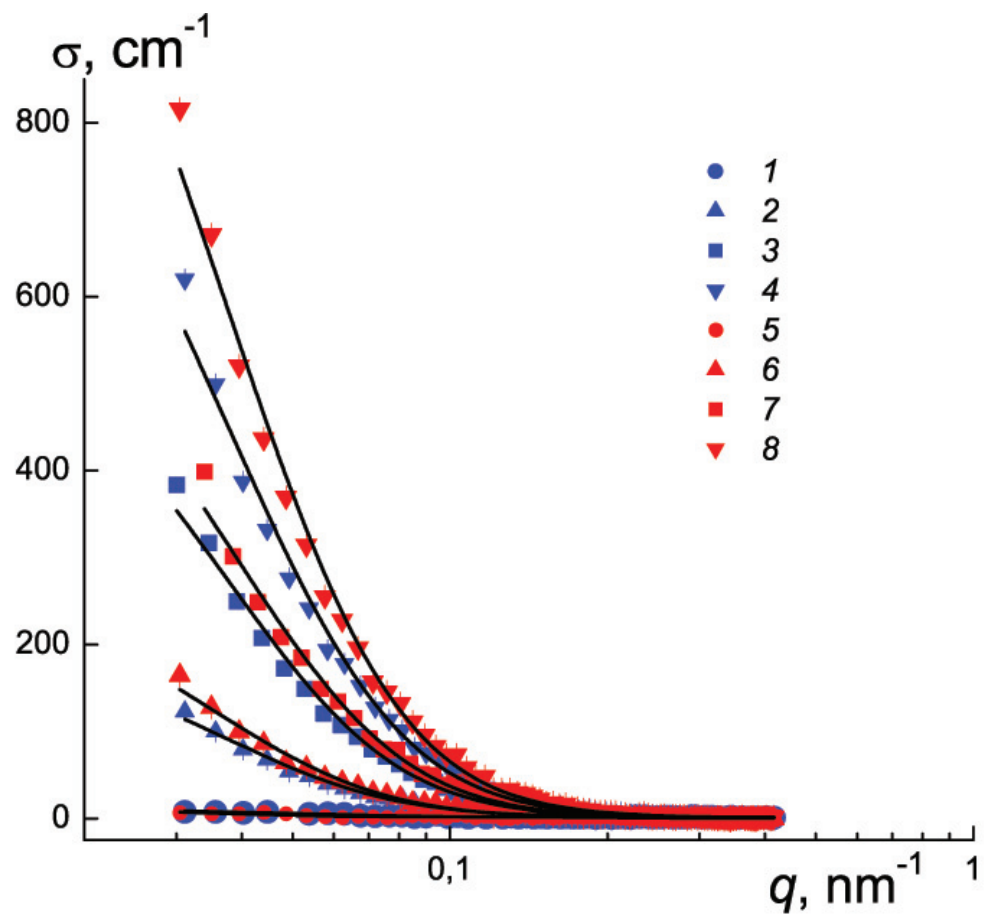

FIG. 1. Scattering cross sections of the solutions with different contents of fullerenols $(0.044,0.056,1.04$ and $2.0 \mathrm{wt} \%)$ studied in weak and strong fields $\left(B_{1}, B_{3}\right)$ (data $1-4$ and $5-8$, respectively). Lines are fitting functions (1)

The application of a magnetic field caused a remarkable increase in the scattering without qualitatively altering the cross section's $q$-dependence, which is clearly visible at concentrations $C=0.056,1.04$ and $2.0 \mathrm{wt} \%$. In the most dilute solution $(C=0.044 \mathrm{wt} \%)$, an opposite situation is observed when the intensity slightly decreases with field application (Fig. 2).

In Fig. 2 the differences of cross section, $\Delta \sigma(q, B)=\sigma(q, B)-\sigma\left(q, B_{1}\right)$, are presented for the dilute system showing a negative effect. Although, in the experimental $q$-range, the application of moderate and strong fields $\left(B_{2}, B_{3}\right)$ does not lead to more intensive scattering, but at the same time, the deficit in cross section means that the scattering redistribution favors lower momentum transfers if the field stimulates aggregation. Indeed, this trend is observed in more concentrated solutions (Fig. 3), where the positive differences of cross sections become greater in the strong field $B_{3}$.

This qualitative analysis has been supplemented by a detailed description of structuring when we used the scattering function : 

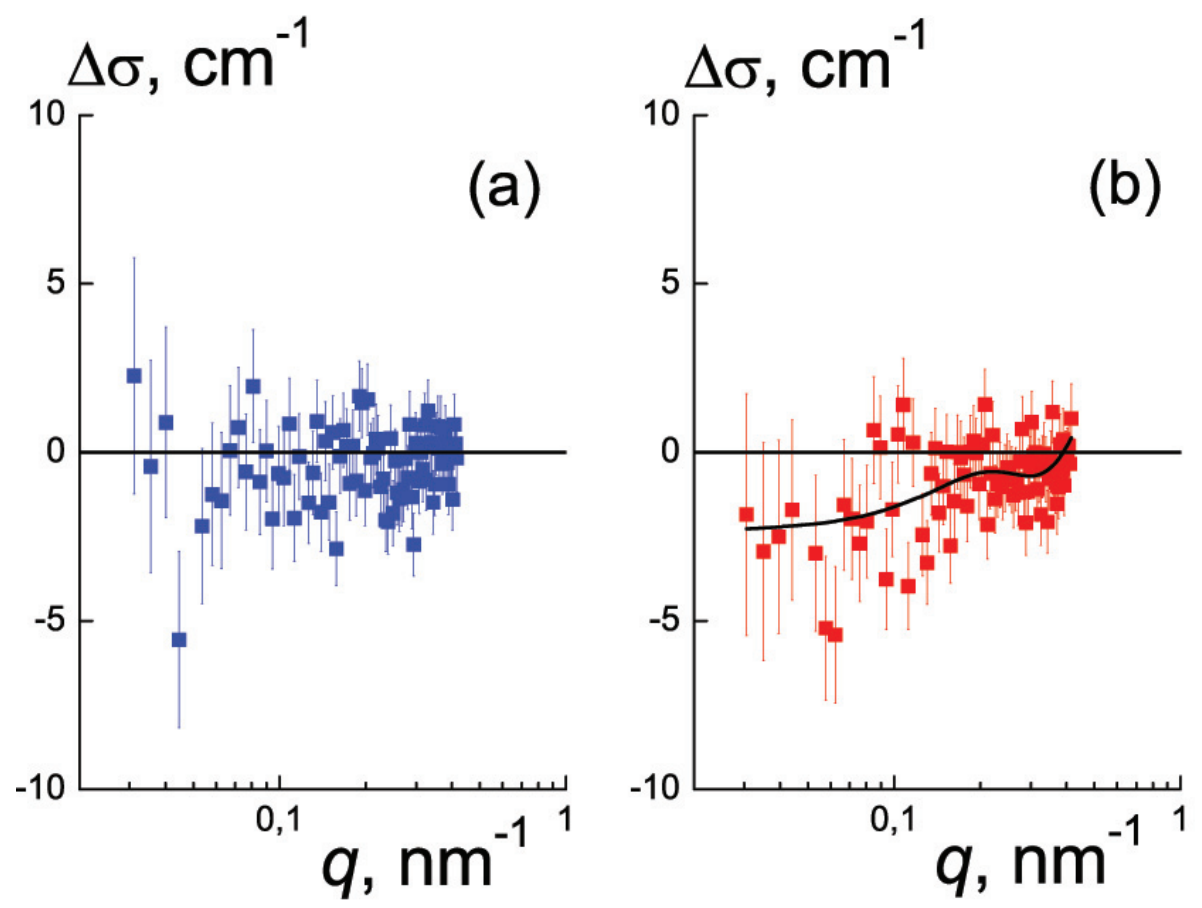

FIG. 2. Differences of cross sections $\Delta \sigma(q, B)=\sigma(q, B)-\sigma\left(q, B_{1}\right)$ in dilute solution $(C=0.044 \mathrm{wt} \%)$ measured in moderate and strong fields $\left(B_{2}, B_{3}\right)(\mathrm{a}$, b) as compared to the data obtained in weak field $B_{1}$. Line (b) shows a splinefunction

$$
\sigma(q)=\frac{\sigma_{0}}{\left[1+\left(q R_{C}\right)^{2}\right]^{2}}+B g
$$

where $\sigma_{0}=\sigma(q \rightarrow 0)$ is forward cross section, $R_{C}$ is the radius of molecular correlations, the parameter $B g$ denotes a contribution of incoherent background to the total cross section. The formalism in (1) satisfactorily fits the experimental data for all concentrations and magnetic field strengths.

The parameters $\sigma_{0}(C), R_{C}(C)$ showed how the behaviors were modified by the external applied magnetic field (Fig. 4). The forward cross sections demonstrate quite linear growth (Fig. 4a) disturbed by quadratic terms, $\sigma_{0}(C)=\left(\alpha_{1} C+\alpha_{2} C^{2}\right)$ where $\alpha_{1}, \alpha_{2}$ are the fitting parameters. Conversely, the parameter $\sigma_{0}=(\Delta K)^{2} \varphi\left(m V_{F}\right)$ is proportional to the aggregation number $(m)$. This also includes the contrast factor of fullerelols in water $\left(\Delta K=4.8 \cdot 10^{10} \mathrm{~cm}^{-2}\right)$, their total volume fraction $\varphi=\left(C N_{A} / M_{F}\right) V_{F}$ which depends on Avogadro's constant $\left(N_{A}\right)$, molecular weight $\left(M_{F}\right)$ and the volume $\left(V_{F}\right)$ of fullerenol molecule. Obviously, at a constant aggregation number $(m)$, there should be linear behavior $\sigma_{0} \sim \varphi \sim C$. This means an increase in the numbers of clusters with the enrichment of solutions. Additionally, the term $\left(\alpha_{2} C^{2}\right)$ arises due to clusters' pair interactions. The increase in the relative rate of aggregation is given by $(1 / m) d m / d C=\alpha_{2} / \alpha_{1}$. This ratio rises more than twice from $\alpha_{2} / \alpha_{1} \approx 9$ to $\approx 19 \mathrm{~cm}^{3} / \mathrm{g}$ due to the induction enhancement from the minimum to the maximum magnetic field strength. Hence, magnetic field stimulates more dense integration of fullerenols when the size of clusters $\left(R_{C}\right)$ remains fairly constant (Fig. 4b).

At magnetic fields ranging from $B=0.01-1.0 \mathrm{~T}$ the correlation radius takes the value $R_{C} \sim 18-19 \mathrm{~nm}$ for the concentrations $C=0.056,1.04$ and $2.0 \mathrm{wt} \%$ (Fig. 4b). However, in weak and moderate fields, at the lowest fullerenol content, $(0.044 \mathrm{wt} \%)$ the correlation lengths 

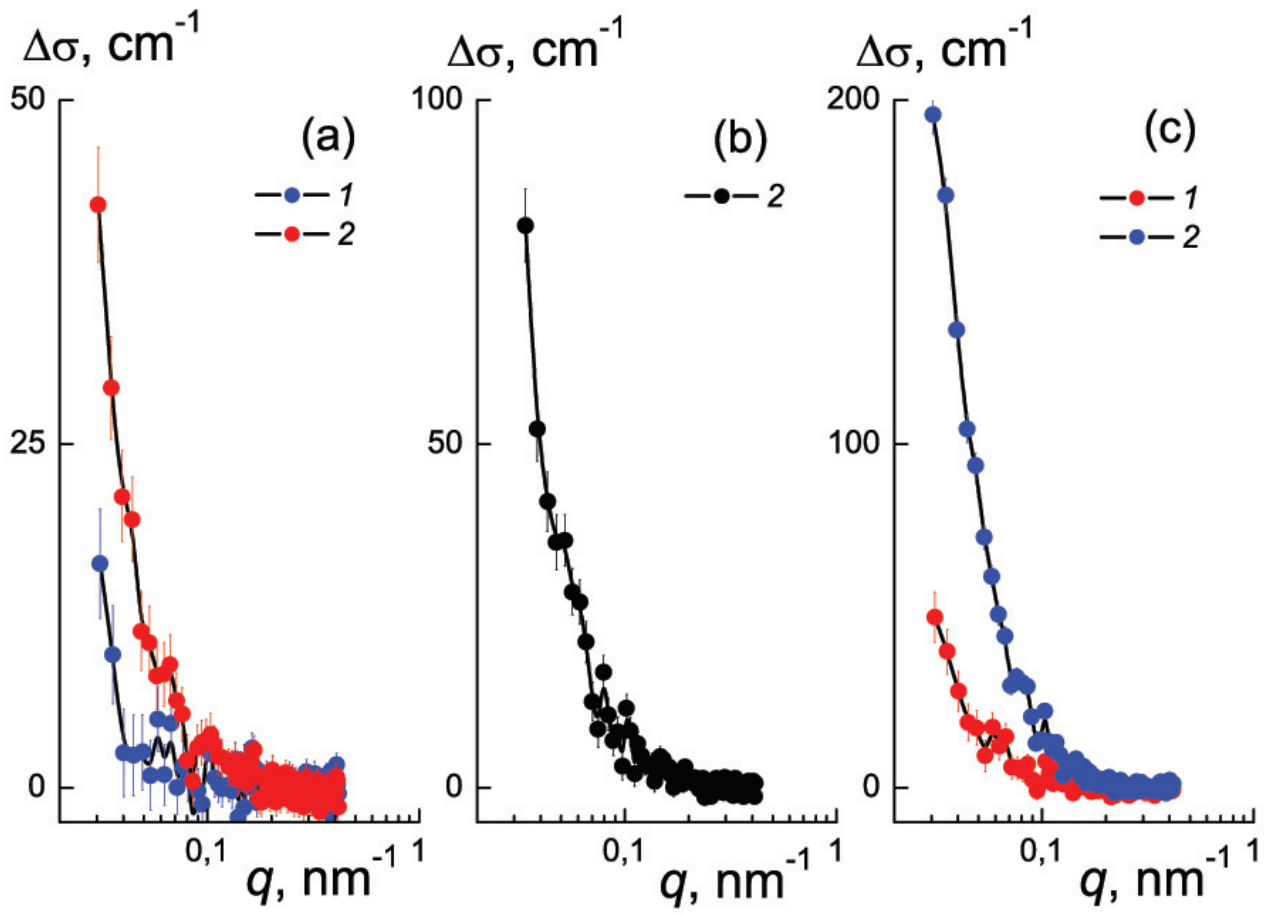

FIG. 3. Field induced gain in cross sections of solutions, $\Delta \sigma(q, B)=\sigma(q, B)-$ $\sigma\left(q, B_{1}\right)$, with fullerenols concentrations $C=0.056,1.04$ and $2.0 \mathrm{wt} \%$ (a, b, c). Data 1, 2 are related to the experiments at moderate and strong fields $\left(B_{2}, B_{3}\right)$

are shorter, $R_{C} \sim 15 \mathrm{~nm}$, while a strong field amplifies the correlations, the radius of which becomes larger, $R_{C} \sim 18 \mathrm{~nm}$ (Fig. $4 \mathrm{~b}$ ). Thus, under the strong field ( $B_{3}=1.0 \mathrm{~T}$ ), clusters achieve the size $R_{C} \sim 18-19 \mathrm{~nm}$ practically not influenced by fullerenols concentrations. Along with this, the aggregation numbers $m(C)$ found from the forward cross sections grow intensively with the concentration, especially in the strong field $B_{3}$ (Fig. 5).

The number of molecules $m \sim(2-5) \cdot 10^{4}$ (Fig. 5) occupies a relatively extended region with gyration radius $R_{G}=\sqrt{6} \cdot R_{C} \sim 40 \mathrm{~nm}$. For instance, a spherical region with such gyration radius has the geometric radius $R_{S}=(5 / 3)^{1 / 2} R_{G} \sim 60 \mathrm{~nm}$. In a spherical approximation, one can estimate the average share of occupied volume, $\Phi \sim 3-7 \%$. Alternatively, a characteristic volume of molecular correlations $V_{C}=8 \pi R_{C}^{3} \sim 2 \cdot 10^{5} \mathrm{~nm}^{3}$ is smaller, and in this region, the occupied volume is of $15-35 \%$. This figure increases with the fullerenol concentration and becomes higher in a strong field, which stimulates the integration of fullerenols into clustered structures.

The analysis performed above concerned the cross sections corresponding to the sum of scattering intensities $I^{+}(q), I^{-}(q)$ obtained by reversing beam polarization $\left( \pm P_{0}\right)$ in vertical guide-field. In general, each intensity [8]:

$$
I^{ \pm}=I_{n}(q)+I_{m}(q) \pm I_{I N T}(q),
$$

includes the contributions of nuclear and magnetic scattering, $I_{n}(q) \sim a_{N}^{2}, I_{m}(q) \sim\left[b_{M} \cdot \sin (\alpha)\right]^{2}$, with the amplitudes $a_{N}(q)$ and $b_{M}(q) \sin (\alpha)$ where $\alpha$ is the angle between induction $\mathbf{B}$ and scattering vector q. In addition, it presents the interference part $I_{I N T}(q) \sim 2 P_{0} a_{N}(q) b_{M}(q \alpha)$, in our experiments $\mathbf{P} \| \mathbf{B}(q)$. This part was extracted as the difference:

$$
\Delta(q)=I\left(q, P^{+}\right)-I\left(q, P^{-}\right) \equiv I^{+}(q)-I^{-}(q)=2 I_{I N T}(q) .
$$



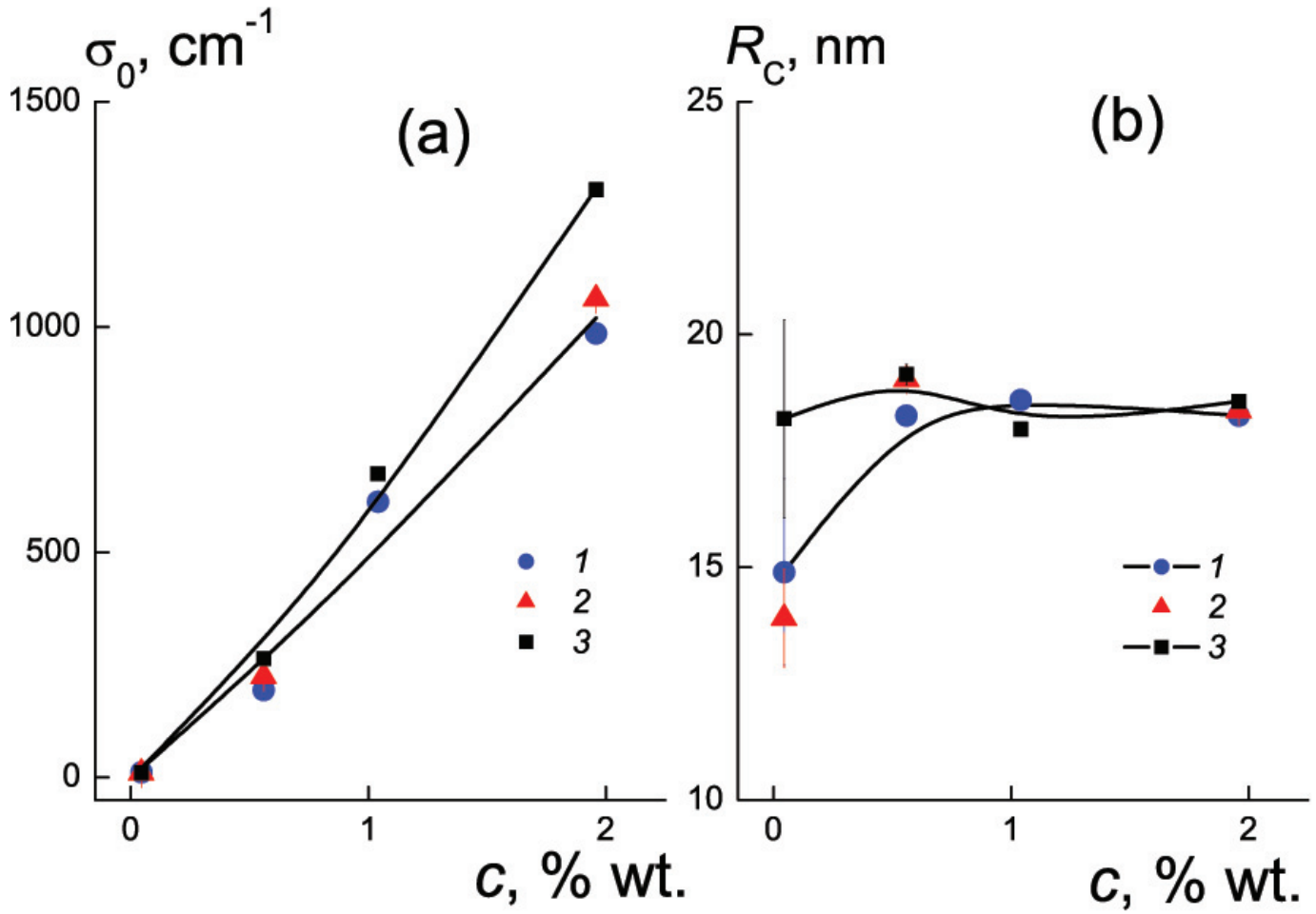

FIG. 4. Concentration and field dependence of the fullerenols clusters parameters: forward cross section $\sigma_{0}(C)$ and correlation radius of clusters $R_{C}(C)(\mathrm{a}, \mathrm{b})$ at external fields $B_{1}, B_{2}, B_{3}$ (data 1-3)

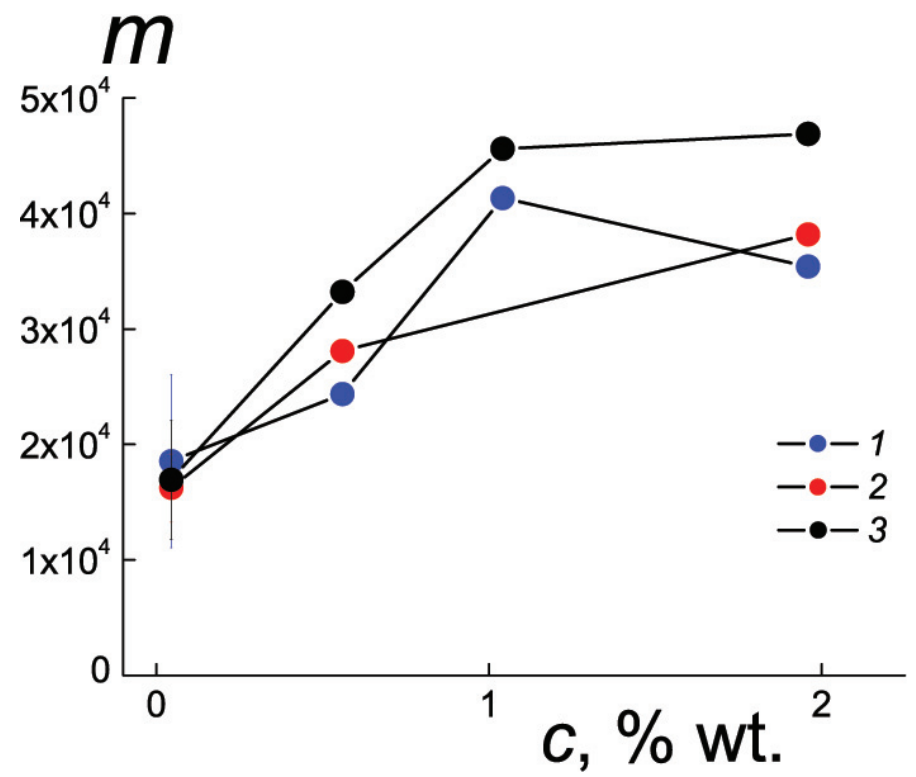

FIG. 5. Aggregation number vs. concentration when magnetic fields $B_{1}, B_{2}, B_{3}$ are applied (data 1-3)

The relative contribution of interference to the total scattering intensity is given by the effective polarization $P_{e f}=\left(I^{+}-I^{-}\right) /\left(I^{+}+I^{-}\right)$. In solutions having weakly magnetic properties, a search of nuclear-magnetic interference has shown low effects averaged over qrange, $\left\langle P_{e f}\right\rangle=(0.07 \pm 0.05) \%$, even at the maximum concentration $C=2.0 \mathrm{wt} \%$ (Fig. 6). 


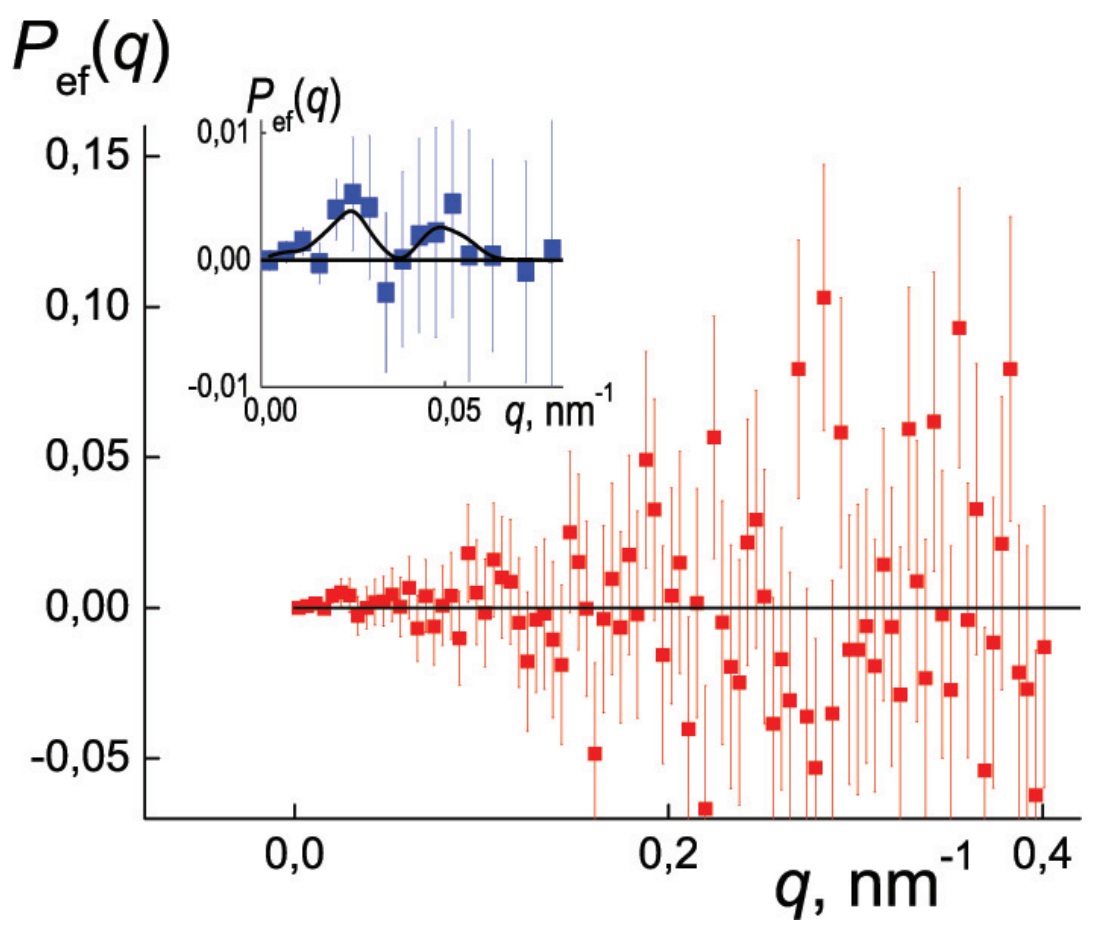

FIG. 6. Effective polarization $P_{e f}(q)$ vs. momentum transfer in fullerenol solutions (concentration $C=2.0 \mathrm{wt} \%$ ). Inset shows the behavior of effective polarization at low $q$-values

This can be explained as a result of low concentration of paramagnetic Gd atoms in these systems, $N_{G d}=2 \cdot 10^{18} \mathrm{~cm}^{-3}$ at $C=2.0 \mathrm{wt} \%$. The applied field $B_{3}=1.0 \mathrm{~T}$ did not align the magnetic moments of Gd. For complete magnetization, higher induction is needed (by two orders in magnitude). This would appear to be in contradiction with the observed substantial amplification of the molecular ordering under magnetic field $B_{3}=1.0 \mathrm{~T}$. Meanwhile, such a discrepancy is eliminated if we consider the induced diamagnetic moments in massive clusters interacting in solutions that stimulates ordering in molecular solutions even at the low diamagnetic susceptibilities of carbon structures $\left(\chi \sim 5 \cdot 10^{-7}\right.$ for $\mathrm{C}_{60}$ fullerite [10]). Indeed, some large-scale effects in effective polarization are visible in the inset shown in Fig. 6 where two maxima are present at $q^{*} \sim 0.025 \mathrm{~nm}^{-1}$ and $q^{* *} \sim 0.05 \mathrm{~nm}^{-1}$. One might suspect that the nuclear-magnetic interference is detected at large scale $R \sim 2 \pi / q^{*} \sim 300 \mathrm{~nm}$ for the superstructures of weakly magnetized clusters.

\section{Conclusions}

The first scattering experiments with polarized neutrons on the mixtures of paramagnetic and diamagnetic fullerenols have revealed unexpected features in fullerenols behavior. A strong applied magnetic field was shown to stabilize the size of molecular aggregates $\left(R_{C} \sim 20 \mathrm{~nm}\right)$ when the concentrations were varied by two orders of magnitude, i.e. the system concentration was changed from very dilute to concentrated (close to the threshold of fullerenol solubility).

The influence of magnetic field does not only involve direct alignment of the paramagnetic atoms' moments. A substantial enhancement of the aggregation numbers was detected under a strong applied magnetic field. In addition, the scattering of polarized neutrons gave specific indications for the correlated spatial organization of molecular and magnetic subsystems at large (submicron) scales where the magnetic domains are visible. 
The fundamental importance of the performed experiments is combined with the practical benefits of the possible application of these substances as effective contrast agents in MRI. As a result of these studies, structural stability has been confirmed for EMF aggregates over a broad range of concentrations in solutions under strong applied magnetic fields.

\section{Acknowledgements}

The work is supported by Russian Found for Basic Research (grant 14-23-01015 ofi_m).

\section{References}

[1] Heath J.R., O'Brien S.C., et al. Lanthanum complexes of spheriodal carbon shells. J. Am. Chem. Soc., 1985, 107, P. 7779-7780.

[2] Piotrovsky L., Eropkin M., et al. Biological Effects in Cell Cultures of Fullerene $\mathrm{C}_{60}$ : Dependence on Aggregation State. In: Medicinal Chemistry and Pharmacological Potential of Fullerenes and Carbon Nanotubes. Eds. F. Cataldo, T. Da Ros. Germany: Springer, 2008, P. 139-155.

[3] Kato H., Kanazawa Y., et al. Lanthanoid endohe-dral metallofullerenols for MRI contrast agents. J. Am. Chem. Soc., 2003, 125, P. 4391-4397.

[4] Yin J.J., Lao F., et al. The scavenging of reactive oxygen species and the potential for cell protection by functionalized fullerene materials. Biomaterials, 2009, 30 (4), P. 611-621.

[5] Grushko Yu.S., Kozlov V.S., et al. MRI-contrasting system based on water-soluble fullerene / Gdmetallofullerene mixture. Fuller., Nanotub. Carbon Nanostr., 2010, 18 (4), P. 417-421.

[6] Grushko Yu.S., Sedov V.P., Kozlov V.S., Tsirlina E.V. Method of preparation of MRI-contrasting agent. Patent RU 2396207 C2 from 10.08.2010, Bull. No. 22, 2010.

[7] Toth E., Bolskar R.D., et al. Water-soluble gadofullerenes: toward high-relaxivity, pH-responsive MRI contrast agents. J. Am. Chem. Soc., 2005, 127 (2), P. 799-805.

[8] Runov V.V., Il'in D.S., Runova M.K., Radzhabov A.K. Study of ferromagnetic correlations caused by the impurities in non magnetic materials by the method of small-angle scattering of polarized neutrons. JETP Lett., 2012, 95 (9), P. 467-470.

[9] Lindner P. Water standard calibration at D11 verified with polymer samples. J. Appl. Crystallography, 2000, 33, P. 807-811.

[10] Kitazawa H., Hashi K., et al. Molecular dynamics and structural phase transition in $\mathrm{C}_{60}$ nanowhiskers. $J$. Phys.: Conf. Series, 2009, 159, 012022 (1). 